Revue internationale P.M.E.

Économie et gestion de la petite et moyenne entreprise

\title{
L'évolution de la structure de financement après un premier appel public à l'épargne: une étude descriptive
}

\section{Josée St-Pierre et Robert Beaudoin}

Volume 8, numéro 3-4, 1995

URI : https://id.erudit.org/iderudit/1008364ar

DOI : https://doi.org/10.7202/1008364ar

Aller au sommaire du numéro

Éditeur(s)

Presses de l’Université du Québec

ISSN

0776-5436 (imprimé)

1918-9699 (numérique)

Découvrir la revue

Citer cet article

St-Pierre, J. \& Beaudoin, R. (1995). L'évolution de la structure de financement après un premier appel public à l'épargne: une étude descriptive. Revue internationale P.M.E., 8(3-4), 181-203. https://doi.org/10.7202/1008364ar
Résumé de l'article

D'après bon nombre d'auteurs ayant étudié les difficultés financières des PME, il semble que celles-ci souffrent d'un problème chronique de sous-capitalisation dû à leur accès limité aux différentes sources de financement offertes en général aux grandes entreprises. Dans cette étude essentiellement descriptive, nous avons voulu examiner des PME à deux stades particuliers de leur développement soit celui de la croissance et celui de l'accès au financement public. Les résultats indiquent que la croissance, la rentabilité et la taille semblent influer sur l'étendue de l'endettement dans le financement total ainsi que sur sa composition. On constate en plus que les PME réduisent leur endettement total au moment de l'accès à l'épargne publique, mais qu'elles l'augmentent dans les années suivantes. Ces quelques résultats préliminaires pourraient permettre de confirmer la théorie de Myers sur l'ordre hiérarchique qui semble convenir parfaitement à la spécificité des PME. 


\title{
L'évolution de la structure de financement après un premier appel public à l'épargne: une étude descriptive
}

\author{
Josée ST-PIERRE* \\ Robert BEAUDOIN** \\ Université du Québec à Trois-Rivières
}

MOTS CLÉS

\section{Appel public à l'épargne - Financement des PME}

Bourse - Écart financier

\begin{abstract}
RÉSUMÉ
D'après bon nombre d'auteurs ayant étudié les difficultés financières des PME, il semble que celles-ci souffrent d'un problème chronique de sous-capitalisation dû à leur accès limité aux différentes sources de financement offertes en général aux grandes entreprises. Dans cette étude essentiellement descriptive, nous avons voulu examiner des PME à deux stades particuliers de leur développement soit celui de la croissance et celui de l'accès au financement public. Les résultats indiquent que la croissance, la rentabilité et la taille semblent influer sur l'étendue de l'endettement dans le financement total ainsi que sur sa composition. On constate en plus que les PME réduisent leur endettement total au moment de l'accès à l'épargne publique, mais qu'elles l'augmentent dans les années suivantes. Ces quelques résultats préliminaires pourraient permettre de confirmer la théorie de Myers sur l'ordre hiérarchique qui semble convenir parfaitement à la spécificité des PME.
\end{abstract}

* Josée St-Pierre est titulaire d'un doctorat en finance (Ph.D.) de l'Université Laval. Elle est l'auteure de plusieurs articles scientifiques dans les domaines de la régie d'entreprises, des offres publiques d'achat et du financement par projet. Ses intérêts de recherche portent également sur l'étude des problèmes financiers des PME. Elle est membre du GREPME depuis 1993. Adresse : Université du Québec à TroisRivières, 3351, boulevard des Forges, C.P. 500, Trois-Rivières (Québec) G9A 5H7.

** Robert Beaudoin est détenteur d'un doctorat en gestion de l'Université AixMarseille III. Ses intérêts de recherche portent principalement sur l'étude des problèmes financiers des PME. Il a œuvré pendant plusieurs années comme cadre supérieur à l'Université du Québec à Trois-Rivières. Il est membre du GREPME depuis 1994. Adresse: Université du Québec à Trois-Rivières, 3351, boulevard des Forges, C.P. 500, Trois-Rivières (Québec) G9A 5H7. 


\section{ABSTRACT}

One of the major financial problems cited in the literature on small and medium size enterprises (SME) is that these one suffer from chronic undercapitalisation due to their limited access to financial markets open to large firms. In this descriptive research, we have considered SME at two specific stages of their life cycle: the growth cycle and the first public offering cycle. Our results indicate that growth, return on investment and size are related to the level of overall indebtedness and to the relative importance of short and long term debt. SME have a lower indebtedness immediately prior to their initial public offering but move back to a higher ratio in the years following the offering. These preliminary results suggest that the Myers pecking order theory on capital structure seems to apply very well to SME financing behavior.

\section{RESUMEM}

Según un número importante de autores que han estudiado las dificultades financieras de las PyMEs, el problema crónico de descapitalización de éstas se debe al acceso limitado a las diferentes fuentes de financiación, ofrecidas en general a las grandes empresas (GE). En este estudio esencialmente descriptivo, hemos querido examinar las PyMEs en dos momentos particulares de su desarrollo, el de crecimiento y el de "financiamiento público". Los resultados demuestran que el crecimiento, la rentibilidad y el tamaño, influyen sobre la importancia del endeudamiento en la financiación total e igualmente sobre su composición. Se comprueba además que las PyMEs reducen su endeudamiento total en el momento de acceder al "ahorro público", pero lo aumentan en los años subsiguientes. Estos resultados preliminares sugieren que la teoría de Myers sobre el orden jerárquico es aplicable al comportamiento en la financiación de las PyMEs.

\section{Introduction}

D'après bon nombre d'auteurs ayant étudié les difficultés financières des $\mathrm{PME}^{1}$, il semble que celles-ci souffrent d'un problème chronique de sous-capitalisation dû à leur accès limité aux différentes sources de financement offertes en général aux grandes entreprises (ci-après GE). Tamari (1980) affirme même que ce phénomène nuit au développement des PME qui doivent recourir à un financement plus coûteux que celui qu'elles pourraient obtenir si elles opéraient dans un marché parfait.

Cet écart dans les possibilités de financement entre des PME et les GE, ainsi que les coûts occasionnés par leur statut de PME, crée ce que les auteurs appellent l'écart financier (finance gap). On définit l'écart financier comme suit: une situation selon laquelle une petite entreprise aurait atteint une étape dans son développement où elle aurait fait un usage maximal des sources de

1. Pour une revue exhaustive de la littérature, voir McMahon et al., 1993. 
financement à court terme, mais n'aurait pas atteint un stade qui lui permette d'accéder au financement à long terme offert par les banques ou par les marchés des capitaux (obligataire ou boursier).

L'écart financier semble avoir deux composantes principales:

- l'écart dû aux connaissances (knowledge gap) : l'utilisation restreinte du financement obligataire est une conséquence directe du manque de connaissances des entrepreneurs concernant les différentes sources de financement, leurs avantages et leurs inconvénients, et les coûts implicites ;

- l'écart dû à l'offre (supply gap): les fonds ne sont pas disponibles pour les PME, ou encore, certaines sources de financement sont disponibles, mais à des coûts bien supérieurs à ceux offerts aux GE.

Voyons au tableau 1 si les différents auteurs ont pu prouver qu'il existait effectivement un écart entre le financement offert aux PME et celui offert aux GE concernant les choix ou les coûts de financement.

Les conclusions proposées par ce tableau ne nous permettent pas d'affirmer, hors de tout doute, qu'il existe un problème dans l'offre de financement globale à l'ensemble des PME. Cela ne nous permet toutefois pas de conclure que les théories financières développées pour expliquer le choix de structure de financement de la grande entreprise pourraient s'appliquer aux PME. Ang (1991) affirme que les coûts élevés d'imperfection des marchés assumés par les PME sont suffisants pour justifier le fait que la gestion financière de ces entreprises de même que leurs choix de mode de financement et de structure de capital reposent sur des éléments différents de ceux des grandes entreprises.

\section{Revue des études empiriques}

Sur un plan conceptuel, Ang (1992) présente un nombre important de facteurs permettant de justifier une forte ou une faible utilisation de l'endettement chez les PME (tableau 2).

On ajoute aux éléments justifiant une faible utilisation de l'endettement les coûts élevés de faillite et d'asymétrie de l'information.

Le problème présumé de sous-capitalisation a donné lieu dans plusieurs pays industrialisés (Canada, Australie, Grande-Bretagne, États-Unis) à des commissions gouvernementales ayant pour but d'étudier ce phénomène et de proposer, le cas échéant, des solutions appropriées. Il convient donc ici de vérifier l'état de la question à partir des résultats de plusieurs études scientifiques (tableau 3). 


\section{TABLEAU 1}

\section{Résumé de quelques études sur l'accès des PME} aux sources de financement

\begin{tabular}{|c|c|c|}
\hline Auteurs & Étude & Conclusions \\
\hline $\begin{array}{l}\text { Bird et } \\
\text { Juttner } \\
\text { (1975) }\end{array}$ & $\begin{array}{c}\text { Enquête } \\
\text { auprès de } \\
1545 \\
\text { entreprises } \\
\text { australiennes }\end{array}$ & $\begin{array}{l}\text { Capital de démarrage : Fourni par le ou les pro- } \\
\text { priétaires. } \\
\text { Refinancement : Fonds propres venant surtout } \\
\text { du réinvestissement des profits. } \\
\text { Financement externe : Marge de crédit bancaire } \\
\text { et crédit commercial. } \\
\text { Les PME n'ont accès qu'à une quantité limitée de } \\
\text { sources de financement. }\end{array}$ \\
\hline $\begin{array}{l}\text { Tamari } \\
(1980)\end{array}$ & $\begin{array}{l}\text { Recension } \\
\text { d'études } \\
\text { empiriques } \\
\text { concernant le } \\
\text { financement } \\
\text { des PME } \\
\text { dans cinq pays }\end{array}$ & $\begin{array}{l}\text { Le ratio fonds propres / dette totale n'est pas } \\
\text { significativement différent entre les PME et les } \\
\text { GE. Le ratio fonds propres / dette à long terme est } \\
\text { plus élevé pour les PME signifiant que les GE ont } \\
\text { un levier financier supérieur. Les PME utilisent } \\
\text { donc plus l'endettement à court terme. } \\
\text { L'endettement à court terme est surtout composé } \\
\text { de crédit commercial. Les emprunts bancaires } \\
\text { sont significativement inférieurs à ceux des GE. }\end{array}$ \\
\hline $\begin{array}{l}\text { Renfrew } \\
\text { (1982) }\end{array}$ & $\begin{array}{c}\text { Étude de } \\
10000 \\
\text { entreprises } \\
\text { sur les années } \\
1968 \text { à } 1978\end{array}$ & $\begin{array}{l}\text { Financement supplémentaire : Venant } \\
\text { directement des propriétaires. } \\
\text { Préférence pour la marge de crédit qui est utilisée } \\
\text { pour le financement des investissements. } \\
\text { Les propriétaires-dirigeants ne semblent pas } \\
\text { insatisfaits des sources de financement que leur } \\
\text { offre le marché des capitaux. }\end{array}$ \\
\hline $\begin{array}{c}\text { Holmes } \\
\text { Kent } \\
(1991)\end{array}$ & $\begin{array}{c}\text { Enquête } \\
\text { auprès de } 391 \\
\text { entreprises } \\
\text { manufacturières } \\
\text { (secteur du } \\
\text { métal) }\end{array}$ & $\begin{array}{l}\text { Leurs résultats ne semblent pas démontrer qu'il } \\
\text { existe une discrimination face aux PME que ce } \\
\text { soit en termes d'offre de financement ou de coût } \\
\text { de financement. Leur échantillon souffre toutefois } \\
\text { du biais des PME survivantes puisqu'on n'y } \\
\text { retrouve aucune entreprise faillie ou en difficultés } \\
\text { financières. }\end{array}$ \\
\hline $\begin{array}{c}\text { Dunstan } \\
\text { et al. } \\
\text { (1992) }\end{array}$ & $\begin{array}{l}\text { Enquête } \\
\text { auprès de } 425 \\
\text { entreprises } \\
\text { de tailles et de } \\
\text { secteurs } \\
\text { industriels } \\
\text { divers }\end{array}$ & $\begin{array}{l}\text { Coût du financement par dettes : Aucune } \\
\text { différence significative sur l'ensemble des } \\
\text { sources de financement bancaire. Une seule } \\
\text { différence existe quant au coût d'une marge de } \\
\text { crédit qui est environ } 7 \% \text { plus élevé pour les PME. } \\
\text { Coût pour la présentation d'une demande de } \\
\text { prêt : Significativement plus élevé pour les PME. } \\
\text { Cette étude souffre du même problème } \\
\text { d'entreprises survivantes que la précédente. }\end{array}$ \\
\hline $\begin{array}{c}\text { Levratto } \\
\text { (1992) }\end{array}$ & $\begin{array}{l}\text { Étude des } \\
\text { statistiques } \\
\text { financières } \\
\text { sur les PME } \\
\text { publiées par } \\
\text { la Banque de } \\
\text { France }\end{array}$ & $\begin{array}{l}\text { Les PME utilisent plus les crédits bancaires à } \\
\text { court terme que les GE, et elles subissent des } \\
\text { conditions débitrices moins favorables que celles } \\
\text { dont profitent leurs homologues de grande taille. }\end{array}$ \\
\hline
\end{tabular}


TABLEAU 2

Facteurs expliquant le recours à l'endettement chez les PME (d'après Ang)

\begin{tabular}{lc}
\hline $\begin{array}{l}\text { Forte utilisation } \\
\text { de l'endettement }\end{array}$ & $\begin{array}{l}\text { Faible utilisation } \\
\text { de l'endettement }\end{array}$ \\
\hline - Relations d'affaires bien développées entre le & - La différence de taux \\
propriétaire-dirigeant et ses créanciers & d'imposition entre les \\
(notamment son banquier) qui peut mener à & propriétaires-dirigeants et les \\
des coûts de financement plus faibles étant & PME. \\
donné une réduction des coûts d'agence et de & - Un désir d'éviter le \\
surveillance pour l'institution prêteuse. & financement externe et le \\
- Absence totale ou partielle du concept de la & contrôle que ces investisseurs \\
responsabilité limitée (normalement dévolue & pourraient exercer sur les \\
aux sociétés par actions) ce qui augmente les & décisions du propriétaire- \\
garanties potentielles pour les prêteurs. Ces & dirigeant \\
garanties peuvent venir de l'entreprise ou du & - Les coûts d'agence inhérents \\
propriétaire-dirigeant. & à la présence de créanciers \\
- Étant donné qu'il y a peu de créanciers, il y a & externes. \\
moins de conflits potentiels dans des cas de & - Le manque de diversification \\
liquidation, réduisant ainsi l'ensemble des & et le risque d'affaires élevé. \\
charges et des pertes pour le prêteur. Cela & - Les coûts élevés de \\
l'amène à augmenter son offre de financement. & surveillance peuvent inciter \\
- Leur propension au risque (contrairement aux & les créanciers à accroître le \\
dirigeants des GE qui sont plutôt averses au & taux d'intérêt. \\
risque) pourrait amener les propriétaires- & - Réduction de la valeur de \\
dirigeants à utiliser au maximum l'effet de & - l'entreprise aux yeux des \\
levier. & autorités fiscales. \\
\hline
\end{tabular}

De plus, plusieurs chercheurs ont tenté de vérifier auprès de dirigeants de PME si ceux-ci étaient préoccupés par leur structure financière.

Scott et al. (1972) ont démontré que 50\% des entreprises interrogées affirmaient être préoccupées par leur ratio d'endettement déterminé en partie par l'expérience passée et le jugement des dirigeants.

Cooley et Edwards (1983) ont demandé à des dirigeants de PME ( $\mathrm{N}=97)$ d'indiquer les facteurs influençant leur propension à utiliser l'endettement pour se financer. L'incertitude concernant la rentabilité future était le facteur le plus important, alors que les restrictions imposées à l'entreprise par les créanciers étaient le facteur le moins important. Apparemment, les entreprises ayant le ratio d'endettement le moins élevé sont celles pour qui la rentabilité est un élément important, alors que les plus endettées sont préoccupées davantage par le chiffre d'affaires. 
TABLEAU 3

Comparaison des structures financières des PME et des GE

\section{Différences significatives}

Holmes et Kent (1991) ont trouvé que les petites entreprises avaient un ratio de dettes / fonds propres de 2,4:1 par rapport aux grandes, de $1,5: 1$.

Remmers et al. (1974) conclut que la taille n'est pas un facteur explicatif du niveau d'endettement aux États-Unis, alors que les PME sont toutes plus endettées que les grandes dans les autres pays (France, Japon, Norvège, Hollande).

Tamari (1980) conclut que les PME sont moins risquées si le levier est mesuré avec l'endettement à long terme. Elles utilisent plus l'endettement à court terme non bancaire.

Pettit et Singer (1985) confirment que les PME sont plus endettées que les grandes.

Osteryoung et al. (1992) observent un endettement supérieur des GE sur les PME, ainsi qu'une plus grande utilisation de l'endettement à court terme par les PME. (Ils utilisent un échantillon de PME privées.)
Aucune différence significative

Dunstan et al. (1992) : aucune différence sur le ratio de dettes sur fonds propres.

Chen et Balke (1979) concluent que ni le secteur industriel ni la taille n'affectent significativement le ratio d'endettement.

Buckland et al. (1989) démontrent que les PME tendent à viser un ratio d'endettement cible, que cette cible n'est pas déterminée selon les caractéristiques de l'entreprise et n'est pas fonction des considérations théoriques habituelles.

Constand et al. (1991) rapportent que l'endettement et la rentabilité sont négativement liées, laissant entendre que les entreprises les plus rentables ont plus recours au financement interne (autofinancement). Ils démontrent également que la structure de l'actif, la taille et la rentabilité sont toutes liées à l'endettement. La structure de l'actif à court terme est fortement liée à l'utilisation de l'endettement à court terme et la structure des actifs immobilisés est liée à l'endettement à long terme. Ils n'ont toutefois qu'un échantillon de 35 PME.

Autant pour la GE que pour la PME, il semble bien que nos connaissances concernant les éléments gravitant autour de la structure financière soient encore bien limitées et il nous apparaît que toute étude visant à déterminer une «structure financière type» pour les PME est vouée à l'échec. 
Comme le mentionnent Pettit et Singer (1985, p. 58) : «Ce qui est évident, c'est que le niveau optimal d'endettement dans les petites entreprises dépend largement des caractéristiques de l'entreprise et de ses dirigeants.»

Levin et Travis renchérissent avec les arguments suivants:

Dans les entreprises privées, la théorie sur la structure financière ne peut que rarement s'appliquer. L'attitude des dirigeants envers le risque personnel - et non les politiques d'établissement de structure financière utilisées par les sociétés ouvertes - détermine le niveau d'endettement acceptable. Le niveau de dette est souvent déterminé, par exemple, par la capacité personnelle du propriétairedirigeant d'assumer le risque financier. Évidemment, cela est dû au fait que les banques utilisent souvent les actifs des propriétaires-dirigeants pour garantir les emprunts de l'entreprise. Les propriétaires qui choisissent d'aller tout de même vers l'endettement doivent comprendre qu'ils augmentent, à ce moment-là, leur risque personnel. (1987, p. 39)

En conséquence, il ne peut y avoir une théorie unique pour expliquer la structure du capital (voir Ang, 1991). Par ailleurs, Paranque affirme que : «[...] il apparaît que le niveau pertinent de fonds propres est à évaluer quasiment au cas par cas, à l'aune du dynamisme des PMI et de leurs capacités à répondre à la demande qui leur est adressée» (1994, p. 186). Les développements théoriques devront donc tenir compte de ce phénomène.

\section{Théories sur la structure du capital adaptées à la PME}

Plusieurs théories ont été proposées afin de comprendre les choix faits par les dirigeants de PME. Celles-ci sont énoncées ci-dessous et ne sont pas nécessairement mutuellement exclusives.

\subsection{La théorie de l'ordre hiérarchique}

Plusieurs études tendent à valider cette théorie dans les PME. Norton, dans une enquête effectuée auprès d'une centaine de dirigeants de PME américaines en croissance, affirme que :

Un examen des réponses fournies par les dirigeants indique que, contrairement à la théorie financière, les éléments liés aux coûts de faillite, d'agence et d'asymétrie de l'information, ont peu (ou pas du tout) d'influence sur le choix de la structure du capital. Plutôt, les responsables financiers semblent adopter une approche de l'ordre hiérarchique (fonds internes avant fonds externes, et financement par fonds propres en dernier recours), tout en étant conscients des conditions du marché et de leurs préférences personnelles. (1991, p. 287) 
Ang (1991) croit que, dans une version de cette théorie modifiée et adaptée à la réalité des PME, une nouvelle contribution des propriétaires serait placée après l'autofinancement, mais bien avant le financement par dettes.

\subsection{L'approche du choix managérial}

Une justification pour cette approche est formulée par Norton (1991) qui affirme que la gestion financière est un domaine d'étude qui chevauche deux disciplines, soit la gestion et la finance. Et jusqu'à présent, les chercheurs en finance ont plutôt ignoré l'aspect gestion dans leurs travaux sur les décisions de structure financière des entreprises. Des progrès dans notre compréhension des choix de financement ne pourront être réalisés que si les chercheurs en finance incorporent à leur modèle des éléments théoriques de la discipline «gestion»; le cas est encore plus probant dans celui des PME, où l'entrepreneur joue un rôle déterminant dans l'entreprise.

Barton et Matthews (1989) ont développé, dans un contexte de choix stratégique, cinq propositions de recherche sur la structure financière des PME. Ces propositions trouvent leur fondement dans le paradigme de la stratégie, c'est-à-dire les valeurs et les aspirations des dirigeants, les circonstances favorables et les menaces de l'environnement, les forces et les faiblesses internes à l'organisation. Voici ces propositions.

1. La propension des dirigeants à accepter un haut niveau de risque affecte le choix d'une structure financière pour leur entreprise. Le niveau de risque avec lequel les dirigeants se sentent à l'aise, particulièrement dans les petites entreprises où le propriétaire-dirigeant doit garantir personnellement les emprunts de l'entreprise, affecte directement la structure de capital.

2. Les objectifs du propriétaire-dirigeant pour son entreprise affectent la structure financière de celle-ci. La théorie financière traditionnelle ne reconnaît que le paradigme selon lequel les dirigeants doivent respecter l'objectif de la maximisation de la richesse des actionnaires. Dans le cas des PME, ce paradigme ne tient plus dans la mesure où le propriétaire-dirigeant est souvent le seul actionnaire. Ses objectifs personnels, qui sont souvent différents de ceux de maximisation de la richesse, viennent donc influencer son choix de financement : objectifs de croissance, de maintien des informations internes confidentielles, de gestion de l'entreprise en toute quiétude, etc.

3. Les propriétaires-dirigeants vont préférer financer leur entreprise par du financement interne plutôt que par des capitaux externes (dettes ou actions). Les dirigeants veulent conserver le contrôle sur leur entreprise, 
sur les décisions qu'ils prennent ainsi que sur la gestion quotidienne. Ils empêcheront alors tout investisseur externe de participer à ces décisions en privilégiant d'abord le financement interne.

4. La propension au risque des dirigeants et les caractéristiques financières des entreprises vont influencer le niveau d'endettement ainsi que les conditions d'octroi de fonds que les créanciers sont prêts à offrir. Les résultats de certaines recherches selon lesquelles les dirigeants des PME ont tendance à ne faire affaires qu' avec quelques institutions financières (souvent une seule) peuvent confirmer cette hypothèse (voir les études de Haines et al., 1991 et Petersen et Rajan, 1994). Il est plus rentable de développer de bonnes relations avec un banquier, lequel finit par comprendre la personnalité du propriétairedirigeant et accepte son processus décisionnel particulier.

5. Les caractéristiques financières de l'entreprise influent sur la capacité du président-directeur de choisir une structure financière appropriée. Cette proposition est également valable pour les grandes entreprises.

On constate le chevauchement de la théorie de l'ordre hiérarchique de Myers et la théorie managériale surtout dans la troisième proposition concernant la séquence des choix de financement.

\subsection{La théorie du cycle de vie de l'entreprise}

Les résultats de plusieurs auteurs appuient la théorie du cycle de vie de l'entreprise. Ang (1991) rationalise ces résultats en affirmant que les PME doivent passer différents stades dans leur évolution vers le statut de grande entreprise. Il ne peut donc y avoir qu'une théorie unique pour expliquer la structure de capital de ces entreprises, mais plutôt une théorie représentative de chaque stade de développement.

La théorie des stades de développement pose qu'à chacun de ceux-ci, l'entreprise fait face à un ensemble de problèmes qui modifient les choix de financement qui lui sont offerts.

Peterson et Shulman (1987) considèrent qu'une réduction des coûts d'agence chez les PME en croissance ainsi qu'une plus grande variété dans les choix de financement leur permettent de développer une structure financière moins coûteuse. Les risques subis par les créanciers sont réduits, ce qui les amènent à diminuer les taux d'intérêt exigés sur les emprunts. Le coût du capital de l'entreprise se trouve alors à diminuer progressivement. Dans leur étude internationale de PME sur douze pays, ils concluent que les coûts de financement (directs et indirects) diminuent avec la taille et l'âge de l'entreprise. 
Il nous semble intéressant d'analyser la structure du capital des PME à deux stades particuliers de leur développement: les années avant un premier appel public à l'épargne qui caractérisent des PME en croissance; les années après cet appel public, associées à un plus grand degré de formalisme, où les PME devraient tendre à ressembler davantage à la grande entreprise, car ces entreprises ont vaincu l'un des grands problèmes présumés des PME: l'accès au marché des capitaux.

\section{Hypothèses de recherche et échantillon}

Cette difficulté à accéder à un éventail de financement diversifié amènerait les PME à se financer surtout par dettes, au détriment des fonds propres, et surtout à court terme étant donné le marché quasi inexistant du financement obligataire à long terme. L'accès à l'épargne publique devrait donc permettre aux $\mathrm{PME}$ en croissance de rééquilibrer leur structure financière et de réduire ainsi leur risque financier.

Considérant les propositions de Peterson et Shulman (1987), les PME en croissance qui accèdent à une plus grande variété de sources de financement devraient mieux équilibrer leur choix de financement entre court et long terme, et entre capitaux propres et dettes.

\subsection{Hypothèses}

S'il est vrai que les PME ont tendance à être plus endettées que les GE à cause d'un accès limité au marché des capitaux, celles qui accèdent au marché boursier devraient réduire considérablement la proportion de la dette dans leur financement.

\section{Hypothèse 1: Le ratio de dettes totales à l'actif total diminue après un premier appel public à l'épargne.}

Par ailleurs, s'il est également vrai que les PME doivent recourir à l'endettement à court terme même pour financer certains projets d'investissement parce qu'elles n'ont pas accès à l'offre de financement obligataire, on peut supposer que celles qui deviennent publiques ne subissent plus ces contraintes quant à l'offre limitée de financement (supply gap). Desroches et Jog (1989) mentionnent que, parmi les avantages de l'appel public à l'épargne, les entrepreneurs reconnaissent que l'image de leur entreprise s'est améliorée auprès de leurs différents partenaires d'affaires et auprès des institutions financières, et qu'ils auraient ainsi plus de facilité à obtenir du financement externe. On devrait alors retrouver une augmentation de l'endettement à long terme au détriment de l'endettement à court terme, lequel est plus coûteux et risqué que le précédent. 


\section{Hypothèse 2: L'importance relative du financement par dettes à court terme diminue après un premier appel public à l'épargne.}

Finalement, une réduction de l'endettement à court terme et global devrait réduire le risque financier des entreprises ayant accédé au marché boursier et assurer une meilleure protection des bailleurs de fonds externes.

\section{Hypothèse 3 : La couverture des frais financiers devrait s'améliorer} après un premier appel public à l'épargne.

\subsection{Les problèmes de mesure de la structure financière}

Ang (1992) soutient qu'expliquer les choix de financement des PME est plus compliqué que dans le cas des GE étant donné les difficultés à mesurer les différentes composantes de la structure et, entre autres, à séparer ce qui devrait réellement être considéré comme de la dette et ce qui devrait appartenir aux fonds propres. Il soulève le problème des postes tels que les «avances des propriétaires » et les «prêts des dirigeants» ou ce qu'il considère comme des "quasi-fonds propres». Les PME qui font un usage élevé des quasi-fonds propres sont considérées comme sous-capitalisées.

Ang (1992) définit les «quasi-fonds propres» comme étant des dettes détenues par des individus ou des institutions qui s'engagent, envers le propriétaire de l'entreprise (1) à ne pas exercer leur droit de mettre l'entreprise en faillite en cas de difficultés financières et (2) à participer au partage des profits lorsque l'entreprise redevient prospère.

D'autres problèmes de mesure de l'endettement ou de la structure financière des PME soulevés par Ang (1992) incluent:

- des difficultés à départager les actifs de l'entreprise de ceux qui appartiennent au propriétaire (utilisés dans l'entreprise ou comme garanties d'emprunts). Il est souvent impossible de séparer les états financiers de la PME de ceux de son propriétaire;

- des difficultés à classer les différents emprunts tels que les prêts faits par des membres des familles, ou ceux qui sont garantis par les actifs du propriétaire-dirigeant ;

- une sous-évaluation de la participation financière réelle des propriétaires, étant donné l'impossibilité de comptabiliser certaines contributions non monétaires telles que le temps, les salaires insuffisants, l'utilisation gratuite de certains actifs du propriétaire-dirigeant qui servent à la production dans l'entreprise. 
Pour ces raisons, Ang (1992) soutient que les ratios d'endettement classiques ne peuvent permettre d'étudier correctement les structures financières des PME.

L'échantillon utilisé dans cette étude devrait poser peu de difficultés de cette nature, étant donné les choix faits par les dirigeants de rendre leur PME publique et donc d'accéder au statut de «future grande entreprise». Les exigences de la Commission des valeurs mobilières du Québec qui régit les activités des sociétés ouvertes font en sorte que les états financiers des entreprises qui ont décidé de s'inscrire en Bourse soient plus conformes à ceux des GE qu'à ceux de la majorité des PME qui préfèrent demeurer fermées et garder leurs informations confidentielles.

\subsection{Description de l'échantillon}

L'échantillon utilisé est composé de $106 \mathrm{PME}$ ayant fait un premier appel public à l'épargne au Québec entre 1984 et 1987. Le nombre de PME ayant demandé l'inscription en Bourse a été considérablement réduit après le krach boursier du mois d'octobre 1987, et ne s'élève, depuis, qu'à quelques-unes par année. Nous avons donc décidé de ne pas mettre à jour notre échantillon afin de ne pas biaiser en faveur d'entreprises qui auraient accédé à l'épargne publique dans des conditions différentes de même que dans un contexte non similaire à celui qui prévalait avant le krach.

Nous disposons des informations comptables sur une période de cinq ans, soit deux ans avant l'émission publique et deux ans après. En raison de l'absence de certaines informations comptables surtout pour les années extrêmes de la période étudiée, l'échantillon total pourra varier entre 77 et 106 observations. Les problèmes d'utilisation des informations comptables dans les PME, étant donné la qualité parfois contestable de celles-ci, devraient être grandement réduits. L'utilisation d'états financiers vérifiés et les différentes législations et réglementations s'appliquant aux sociétés ouvertes ou sur le point de l'être devraient réduire les difficultés à interpréter les informations des PME faisant partie de l'échantillon.

Étant donné que les hypothèses énoncées devraient nous permettre de vérifier les modifications de structure financière à la suite d'un premier appel à l'épargne publique, nous n'avons nullement tenu compte des effets de secteur dans cette étude. Les entreprises de l'échantillon appartiennent à différents secteurs industriels allant du manufacturier à l'entreprise de service. Nos conclusions ne seront pas touchées par cette disparité dans les secteurs industriels. 


\section{Analyse des résultats}

Cette étude étant uniquement descriptive, nous n'essaierons nullement de vérifier les éléments expliquant les modifications de structures financières, mais plutôt comment celles-ci ont évolué après un premier appel public à l'épargne (APE). À l'aide du logiciel SAS, nous avons tenté de vérifier nos hypothèses seulement à partir de statistiques univariées.

\subsection{Quelques statistiques descriptives}

Le tableau 4 démontre que l'échantillon est composé d'entreprises qui ont connu une croissance moyenne annuelle de leur actif d'environ $55 \%$ au cours des cinq années de la période étudiée, la croissance la plus forte étant évidemment au moment de l'APE. Ces entreprises ont vite atteint le stade de ME après leur inscription en Bourse.

TABLEAU 4

Quelques données descriptives de l'échantillon

\begin{tabular}{|c|c|c|c|c|c|c|c|c|c|c|}
\hline \multirow[b]{2}{*}{ Actif $(000 \$)$} & \multicolumn{2}{|c|}{$\begin{array}{c}2 \text { ans avant } \\
(\mathrm{N}=81)\end{array}$} & \multicolumn{2}{|c|}{$\begin{array}{l}1 \text { an avant } \\
(\mathrm{N}=105)\end{array}$} & \multicolumn{2}{|c|}{$\begin{array}{c}\text { APE } \\
(N=106)\end{array}$} & \multicolumn{2}{|c|}{$\begin{array}{l}1 \text { an après } \\
(N=103)\end{array}$} & \multicolumn{2}{|c|}{$\begin{array}{c}2 \text { ans après } \\
(\mathrm{N}=77)\end{array}$} \\
\hline & $\begin{array}{l}\text { Moy. } \\
7717\end{array}$ & $\begin{array}{r}\text { Méd } \\
5482\end{array}$ & $\begin{array}{l}\text { Moy. } \\
9775\end{array}$ & $\begin{array}{l}\text { Méd } \\
6681\end{array}$ & $\begin{array}{l}\text { Moy. } \\
19407\end{array}$ & $\begin{array}{r}\text { Méd } \\
15826\end{array}$ & $\begin{array}{l}\text { Moy. } \\
28370\end{array}$ & $\begin{array}{r}\text { Méd } \\
21206\end{array}$ & $\begin{array}{l}\text { Moy. } \\
48460\end{array}$ & $\begin{array}{r}\text { Méd } \\
26292\end{array}$ \\
\hline Ventes $(000 \$)$ & 12591 & 8662 & 14056 & 9996 & 21709 & 18197 & 32881 & 27693 & 47508 & 33487 \\
\hline Rendement sur l'actif (\%) & 30,26 & 13,79 & 47,63 & 16,36 & 11,96 & 12,16 & 8,36 & 10,56 & 8,28 & 8,04 \\
\hline Rendement sur l'avoir (\%) & 46,32 & 24,16 & 452,76 & 28,42 & $-40,82$ & 10,82 & $-73,31$ & 10,21 & 0,39 & 6,36 \\
\hline Marge d'exploitation (\%) & 4,56 & 6,23 & 20,88 & 9,66 & 10,47 & 8,87 & 7,48 & 6,71 & 4,13 & 4,18 \\
\hline Marge nette $(\%)$ & $-18,94$ & 4,32 & 1,82 & 6,15 & 4,57 & 5,59 & 3,78 & 4,09 & $-14,93$ & 1,87 \\
\hline Immobilisation / Actif (\%) & 32,26 & 28,85 & 29,06 & 24,99 & 27,44 & 23,07 & 29,40 & 26,40 & 33,55 & 29,16 \\
\hline Actif c.t. / Passif c.t. & 1,51 & 1,32 & 1,60 & 1,34 & 2,70 & 1,89 & 2,26 & 1,60 & 1,95 & 1,68 \\
\hline
\end{tabular}

Étant donné l'asymétrie des distributions, nous utiliserons les valeurs médianes pour formuler les commentaires suivants.

La croissance moyenne (sur les valeurs médianes) des ventes et de l'actif est supérieure à $40 \%$ par année. Cela confirme la qualité de forte croissance des activités des entreprises de l'échantillon.

Les taux de rendement sur l'actif et sur l'avoir subissent une détérioration après l'APE étant donné 1) la forte croissance de l'actif et de la capitalisation et 2) la réduction des marges bénéficiaires nettes et d'exploitation.

Dans les prospectus d'émission, plus du tiers des entreprises avaient indiqué vouloir accéder au marché boursier pour améliorer leur fonds de roulement. On constate effectivement une amélioration du ratio de fonds de roulement (Actif c.t./Passif c.t.) au moment de l'APE et après. 
Par ailleurs, la croissance des immobilisations après l'APE ainsi qu'une réduction des écarts entre le rendement sur l'actif et le rendement sur l'avoir, ce dernier étant même inférieur au précédent deux ans après l'APE, laissent supposer que la forte croissance a donné lieu à un effet de levier négatif pour plusieurs entreprises. Cette situation peut s'expliquer par une forte croissance ou par un appel public à l'épargne prématuré pour certaines d'entre elles, ce qui les amène à investir dans des projets à VAN négative, étant donné leur surplus de liquidités.

\section{2. Évolution de la structure de financement}

Avant l'APE, on retrouvait quatorze rubriques composant la structure financière des entreprises. Les principaux postes étaient évidemment le passif à court terme, qui représentait en moyenne plus de $85 \%$ de l'actif deux ans avant l'APE (par rapport à une valeur médiane de $45 \%$ ). Tout comme l'avait stipulé Ang (1991), ce passif à court terme était gonflé par des «avances des propriétaires» ou des «quasi-fonds propres». Une fois corrigé, le passif à court terme moyen ne représente plus que $53 \%$ de l'actif, comparativement à $29 \%$ pour l'avoir des actionnaires. La figure 1 indique comment les principales sources de financement ont évolué après l'APE. On peut constater que la réduction de l'endettement débute un an avant l'APE, ce qui laisserait présumer que les entreprises commencent à réduire leur risque financier et tentent de «normaliser» leur structure financière avant de devenir publiques de façon à améliorer leurs chances de réussir leur inscription en Bourse et de réduire ainsi le problème de sous-évaluation initiale constaté par plusieurs chercheurs (voir Tinic, 1988).

La lecture des données nous a permis de constater la disparition des postes «avances des propriétaires» ou «prêts des actionnaires» de la dette (court et long terme) après l'inscription en Bourse. On retrouvait ce poste chez $17 \%$ des PME deux ans avant l'APE, $27 \%$ un an avant, $13 \%$ au moment de l'APE, $6 \%$ un an après et $1 \%$ deux ans après. Cette inscription s'est faite en grande partie par une émission d'actions ordinaires et parfois, avec des actions subalternes. Ces dernières ne représentaient toutefois que $2,6 \%$ de l'actif total en moyenne ( $0 \%$ en médiane).

Globalement, le financement par dettes ne représente donc plus que $23,9 \%$ du financement total après un appel public à l'épargne comparativement à $34,8 \%$ deux ans avant, confirmant ainsi notre première hypothèse.

Le phénomène qui nous paraît le plus intéressant est attribuable à l'évolution de la dette à long terme. En effet, on constate son importance deux ans avant l'APE, une réduction sensible au moment de l'APE et, finalement, une 
augmentation deux ans après l'APE où l'on retrouve un niveau similaire au niveau précédent. La réduction de son importance relative dans les années précédant l'APE et au moment de l'émission publique appartient au fait qu'elle a crû moins rapidement que l'actif pendant cette période alors que c'est l'inverse après. Ce résultat pourrait confirmer que les PME nouvellement inscrites bénéficient d'une plus grande offre de financement et que, tout comme l'avait prescrit Myers selon l'ordre hiérarchique, leur croissance serait financée par autofinancement et par dettes plutôt que par d'autres émissions de capital-actions. Ce choix permettrait également une réduction du coût moyen pondéré du capital.

FIGURE 1

Évolution des principales sources de financement des PME ayant accédé au financement public

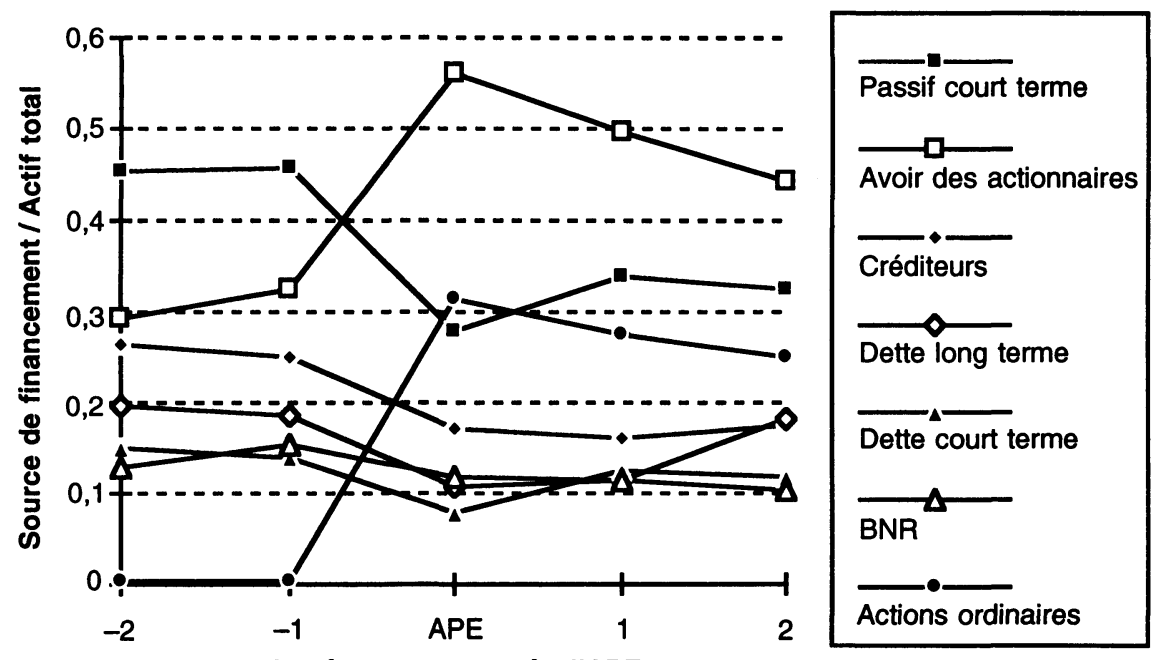

Année avant ou après l'APE

La réduction du passif à court terme est notable, ce qui confirme notre deuxième hypothèse. Tous les postes du passif à court terme ont évolué à la baisse, et on constate la disparition du poste "prêt à court terme» qui est composé uniquement d'avances ou de prêts des propriétaires. La dette à court terme a d'abord connu un mouvement à la baisse pour s'accroître à nouveau après l'APE, demeurant tout de même de $22 \%$ inférieur à son niveau initial. De leur côté, les créditeurs ont diminué de façon importante au moment de l'APE et sont demeurés à ce niveau par la suite. Il semble donc que non seulement le financement à court terme soit relativement moins important après une 
inscription en Bourse mais, en plus, que sa composition se modifie. Tamari (1980) soutient que les propriétaires-dirigeants préféraient le financement des fournisseurs au financement bancaire à cause de sa souplesse et de la noningérence des fournisseurs dans les décisions de l'entreprise. Ce phénomène semble donc se modifier considérablement après un APE.

Finalement, selon la troisième hypothèse, une réduction du risque financier intervenait après un APE. Les données du tableau 5 ne permettent pas de confirmer cette hypothèse, si l'on utilise le ratio conventionnel de couverture des intérêts.

\section{TABLEAU 5}

Couverture des intérêts et risque financier

\begin{tabular}{lrrrrrrrrrrr}
\hline & \multicolumn{2}{c}{ 2 ans avant } & \multicolumn{2}{c}{$\mathbf{1}$ an avant } & \multicolumn{2}{c}{ APE } & \multicolumn{2}{c}{$\mathbf{1}$ an après } & \multicolumn{2}{c}{2 ans après } \\
& Moy. & Méd. & Moy. & Méd. & Moy. & Méd. & Moy. & Méd. & Moy. & Méd. \\
\hline $\begin{array}{l}\text { Intérêt / Passif c.t. + } \\
\text { Dettes i.t. }\end{array}$ & 0,0637 & 0,0624 & 0,1524 & 0,0583 & 0,0612 & 0,0512 & 0,0466 & 0,0437 & 0,0589 & 0,0533 \\
$\begin{array}{l}\text { (Bénéfice imposable } \\
\text { + Intérêts)/ Intérêts }\end{array}$ & 9,173 & 2,9091 & 6,0293 & 4,5736 & 8,1869 & 5,2554 & 11,5969 & 4,6760 & 4,0424 & 2,4749 \\
\hline
\end{tabular}

Le ratio de couverture des intérêts s'est sensiblement détérioré après l'APE. Toutefois, on constate que la part des intérêts ne s'est pas accrue de façon notable par rapport au financement par dettes pendant la période. Au demeurant, on constate une légère diminution qui pourrait probablement indiquer une réduction du coût moyen de financement par dettes, compte tenu que la structure de financement à court terme avant l'APE renfermait surtout des éléments de financement n'entraînant aucune charge financière directe (comptes fournisseurs, éléments à payer, etc.). Ces résultats pourraient donc nous amener à conclure que la couverture des intérêts ne s'est pas détériorée pour les prêteurs après l'APE.

Il semble donc que nous ayons vérifié les deux premières hypothèses énoncées plus haut et que nous ne puissions rejeter la troisième. Dans un deuxième temps, nous avons voulu vérifier si, lorsqu'on n'observe que les PME qui se sont inscrites en Bourse, la structure de financement est la même pour l'ensemble des entreprises et si l'échantillon est relativement homogène. Nous présentons ces résultats dans la prochaine section, après quoi, nous formulerons nos conclusions.

\subsection{Taille, croissance, rentabilité et structure financière}

\subsubsection{Effet de taille}

Plusieurs auteurs mentionnent que la taille est un élément important de la composition de la structure financière des PME (Peterson et Shulman, 1987; 
et voir tableau 3). Nous avons voulu vérifier s'il en était ainsi lorsqu'on séparait nos entreprises en groupes de taille homogène, en les divisant selon les écarts interquartiles de la distribution de l'actif total un an avant l'APE 2 . Nous avons ainsi obtenu quatre groupes d'observations pour lesquelles nous avons mesuré 1) la part du financement à court terme par rapport à l'actif total et 2) la proportion de la dette à long terme par rapport à l'actif total.

Les figures $2 \mathrm{a}$ et $2 \mathrm{~b}$ nous indiquent quelques différences.

On constate que les entreprises les plus petites faisaient, deux ans avant l'APE, un usage plus grand des dettes à court terme (corrigé pour les avances des propriétaires), et que ces différences s'atténuent avec les années. Il semble y avoir un effet de taille où l'on constate qu'à mesure que les entreprises croissent, elles se financent moins par dettes à court terme avant et après l'APE. Lorsqu'on regarde du côté de l'endettement à long terme, on constate un effet de taille inversé. Les PME les plus grandes ont plus recours à la dette à long terme deux ans avant et moins après l'APE, tout comme le signalaient Constand et al. (1991). Elles sont globalement moins endettées que les plus petites après le financement public. Peut-on confirmer que les plus petites entreprises sont plus dépendantes du financement à court terme que les plus grandes?

\subsubsection{Effet de croissance}

Lorsque nous avons regroupé les entreprises selon leur taux de croissance moyen sur l'ensemble de la période (Var Act pour variation de l'actif), nous constatons que celles qui croissent le plus rapidement étaient plus endettées à court terme que les autres (probablement surtout à cause des comptes fournisseurs), et que cette croissance élevée a des effets même deux ans après l'inscription en Bourse pour le long terme. Les PME en plus forte croissance ont substituté une partie de leurs dettes à court terme pour du long terme.

Les figures $3 \mathrm{a}$ et $3 \mathrm{~b}$ nous montrent bien que la croissance semble jouer sur le financement. Cela est évident surtout dans la figure $3 \mathrm{~b}$ où les entreprises qui ont la plus forte croissance ont nettement plus recours à la dette à long terme que celles qui ont une croissance plus faible, ce qui peut s'expliquer par des besoins accrus en immobilisations et donc, en crédits bancaires à long terme.

\subsubsection{Effet de rentabilité}

Finalement, est-ce que la structure de financement est différente selon la rentabilité de l'entreprise? Selon Myers, on devrait s'attendre à observer

2. Nous avons utilisé un an pour diviser notre échantillon plutôt que deux ans avant l'APE, étant donné le nombre d'observations manquantes pour cette année. 
FIGURE 2

Étendue du financement par dettes selon la taille des entreprises

a) Passif à court terme selon la taille

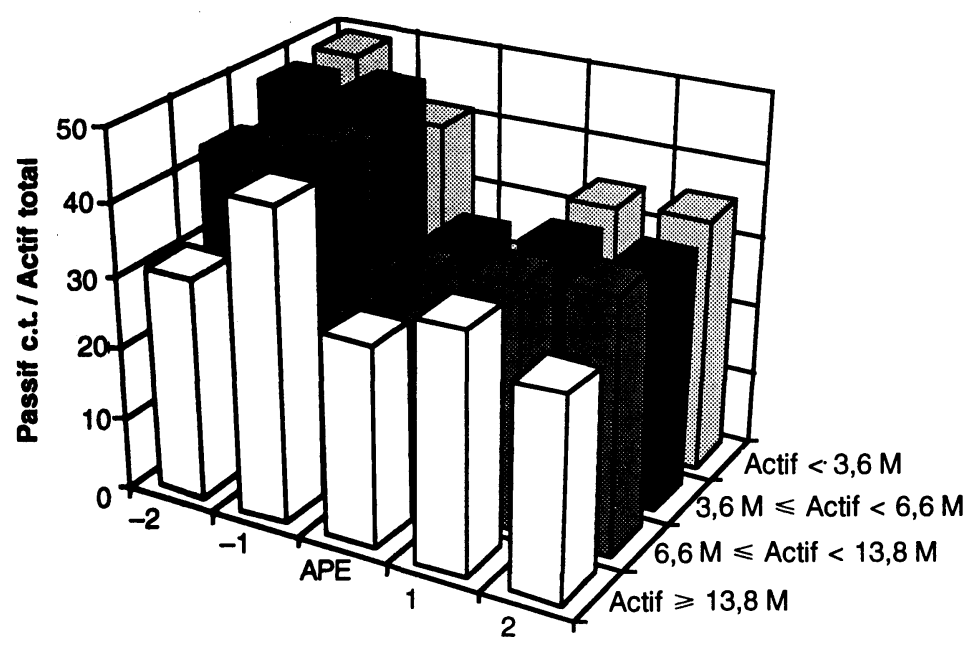

Année avant ou après l'APE

b) Dette à long terme selon la taille

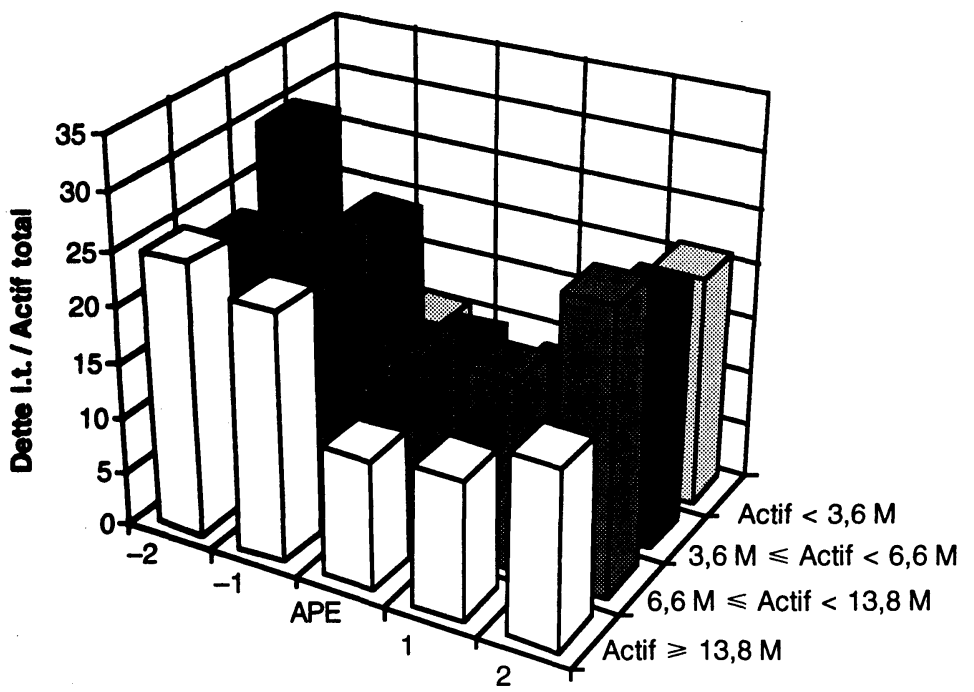

Année avant ou après l'APE 


\section{FIGURE 3}

Part du financement par dettes selon le taux de croissance des entreprises

a) Passif à court terme selon la croissance de l'actif

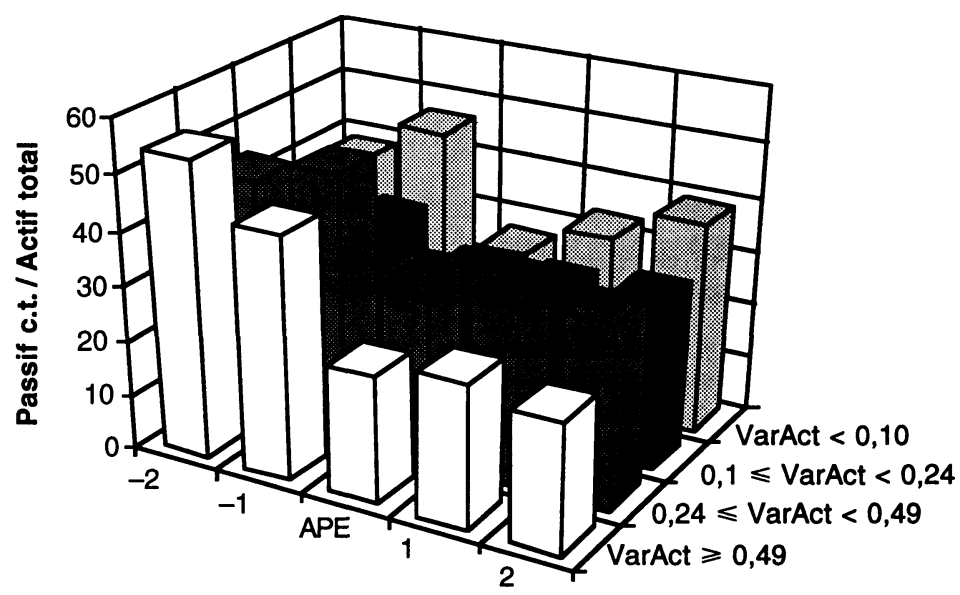

Année avant ou après l'APE

b Dette à long terme selon la croissance de l'actif

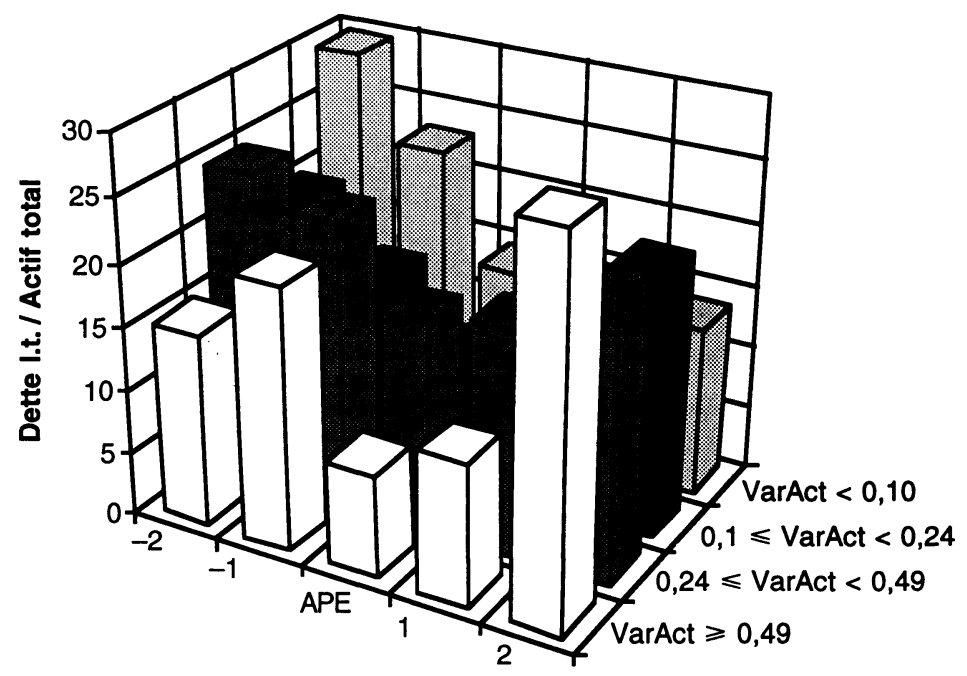

Année avant ou après l'APE 
FIGURE 4

Part du financement par dettes selon la rentabilité des entreprises

a) Passif à court terme selon la rentabilité

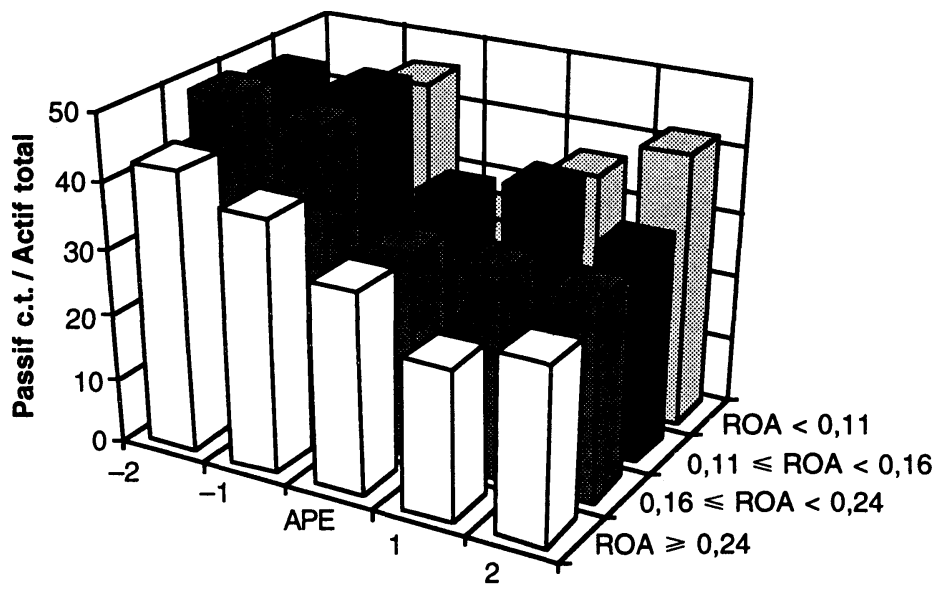

b) Dette à long terme selon la rentabilité

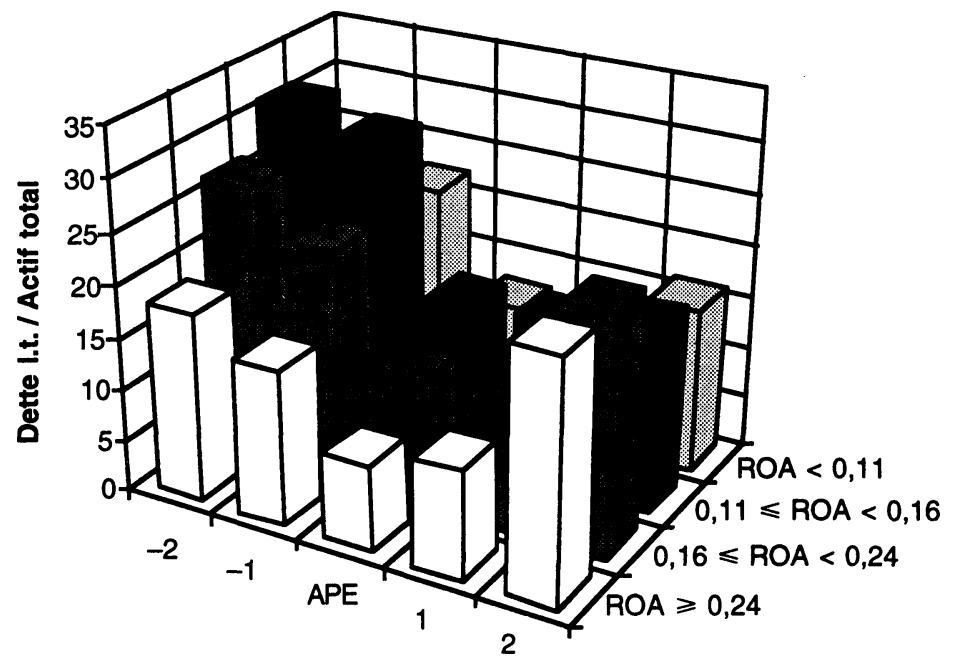


effectivement une différence dans la mesure où les entreprises les plus rentables peuvent davantage recourir à l'autofinancement. D'après la figure 4, il semble que, globalement, les PME les plus rentables (ROA pour taux de rendement de l'actif) soient moins endettées à court terme que les autres après l'APE, alors qu'elles le sont plus à long terme. La faible rentabilité des entreprises du premier groupe peut limiter leur accès au marché des capitaux, de sorte qu'elles sont contraintes à utiliser surtout de l'endettement à court terme. Ces résultats sont différents de ceux de Cooley et Edwards (1983) et Constand et al. (1991); le fait que le classement des entreprises ait été effectué sur la base de la rentabilité avant l'APE peut expliquer ce résultat.

Les différences entre les groupes d'entreprises selon la rentabilité ne sont pas nettes sauf en ce qui concerne les plus rentables qui, globalement, sont moins endettées à court et long terme, et donc moins risquées que les autres.

\section{Conclusion}

Cette étude confirme que l'APE permet aux entreprises une réduction sensible de leur niveau d'endettement. Par contre, on constate qu'après l'inscription en Bourse, l'endettement à long terme augmente à nouveau, ce qui nous amène à penser que l'offre de financement des PME est peut-être sensible au statut d'entreprise fermée ou ouverte, de telle sorte que certaines sources de financement inaccessibles aux plus petites sont rendues accessibles aux entreprises ouvertes. S'agit-il d'un effet de statut ou de taille? Nous ne pouvons y répondre à la lumière des résultats présentés ici. Mais d'après les conclusions de Desroches et Jog (1989), il semble que le statut ait un effet important sur l'accessibilité à certaines sources de financement plus souvent offertes aux GE.

On observe en plus que les entreprises semblent revenir au financement par dettes après l'APE afin, croit-on, de bénéficier d'une réduction de leur coût du capital qui peut être attribuable à des effets fiscaux, à une diminution des coûts d'agence ou de l'asymétrie de l'information. Nos résultats confirment les hypothèses de Myers (1984) selon lesquelles les entreprises préfèrent l'endettement à une augmentation du capital-actions pour financer leur croissance.

Finalement, la taille, le rythme de croissance et la rentabilité semblent influencer la part relative de l'endettement ainsi que la forme de celui-ci. 


\section{Bibliographie}

ANG, J.S. (1991), «Small business uniqueness and the theory of financial management», The Journal of Small Business Finance, vol. 1, no 1, p. 1-13.

ANG, J.S. (1992), «On the theory of finance for privately held firms», The Journal of Small Business Finance, vol. 1, $\mathrm{n}^{0} 3$, p. 185-203.

BARTON, S.L. et C.H. MATTHEws (1989), «Small firm financing: implications from a strategic management perspective», Journal of Small Business Management, janvier, p. 1-7.

BIRD, R. et D. JUTNER (1975), «The financing of small business in manufacturing sector », Cahier de recherche, $\mathrm{n}^{\circ} 69$, Sydney, School of Economic and Financial Studies, Macquarie University.

Buckland, R., E.W. Davis et K.A. Yeomans (1989), «Debt management by companies entering the unlisted securities market», Accounting and Business Research, vol. 19, $\mathrm{n}^{0} 74$, p. 99-111.

Chen, K. et T. BALKe (1979), «Scale of operation, industry and financial ratios», International Journal of Accounting, vol. 14, $\mathrm{n}^{\circ} 2$, p. 17-28.

CONSTAND, R.L., J.S. OSTERYOUNG et D.A. NAST (1991), «Asset-based financing and the determinants of capital sructure in the small firm », dans R. Yazdipour (éd.), Advances in Small Business Finance, p. 29-45.

CoOley, P.L. et C.E. EdWARDS (1983), «Financial objectives of small firms», American Journal of Small Business, vol. 8, $\mathrm{n}^{\circ} 1$, p. 27-30.

DesRocheS, J.Y. et V.M. JoG (1989), «Les effets du financement public sur les entrepreneurs et leurs entreprises: le cas des PME au Québec», Revue Internationale PME, vol. 2, $\mathrm{n}^{\circ} 1$, p. 19-36.

DunSTAN, K., D. DWyER et S. Holmes (1992), «The small business funding debate: an empirical review», International Research Symposium on Small Firm Finance, Baylor University, Texas.

HaINES, G., A. RIDING et R. Thomas (1991), «Small business bank shopping in Canada», Journal of Banking and Finance, vol. 15, p. 1041-1056.

Holmes, S. et P. KENT (1991), «An empirical analysis of the financial structure of small and large Australian manufacturing enterprises», The Journal of Small Business Finance, vol. 1, n ${ }^{\circ} 2$, p. 141-154.

LEVIN, R.I. et V.R. TRAVIS (1987), "Small company finance: what the books don't say », Harvard Business Review, novembre-décembre, p. 30-32.

LeVratto, N. (1992), «L'analyse du financement des PME: une mise en relation du coût des ressources et de la notion de territoire financier», Revue d'Économie Régionale et Urbaine, vol. 2, p. 257-278. 
MCMahon, R.G.P., S. Holmes, P.J. Hutchinson et D.M. ForSAITH (1993), Small Enterprise Financial Management: Theory and Practice, Sydney, Australie, Hartcourt Brace, 488 pages.

MYERS, S.C. (1984), «The capital structure puzzle», The Journal of Finance, vol. 39, $\mathrm{n}^{\circ} 3$, p. 575-592.

NORTON, E. (1991), «Capital structure and small growth firms», The Journal of Small Business Finance, vol. 1, n ${ }^{\circ} 2$, p. 161-177.

OSTERYOUNG, J., R.L. CONSTAND et D. NAST (1992), «Financial ratios in large public and small private firms", Journal of Small Business Management, juillet, p. 35-46.

PARANQUE, B. (1994), «Fonds propres, rentabilité et efficacité chez les PMI : méthodes d'analyse et appréciation des situations financières ", Revue d'Économie Industrielle, vol. 67, $\mathrm{n}^{0} 1$, p. 175-190.

Peterson, R. et J. Shulman (1987), «Capital structure of growing small firms: a 12 country study on becoming bankable», International Small Business Journal, vol. 5, no 4, p. 10-22.

PETERSEN, M.A. et R.G. RAJAN (1994), "The benefits of lending relationships: evidence from small business data», The Journal of Finance, vol. 49, $\mathrm{n}^{\circ} 1$, p. 3-37.

PETTIT, R.R. et R.F. SINGER (1985), «Small business finance: a research agenda», Financial Management, automne, p. 47-60.

Remmers, L., A. Stonehill, R. Wright et T. BeEkHUiSEN (1974), «Industry and size as debt ratio determinants in manufacturing internationally », Financial Management, vol. 36, $\mathrm{n}^{\circ}$ 3, p. 879-888.

RENFREW, L. (1982), «The adequacy of finance for small business in Australia», dans W. Dunlop et W. Sheehan (éd.), Small Business Research Series $n^{0} 11$, Newcastle, Australie, Institute of Industrial Economics, p. 103-108.

SCOTT, D.F., O.L. GRAY et M.M. BIRD (1972), «Investing and financing behavior of small manufacturing firms », MSL Business Topics, vol. 20, n 3, p. 29-38.

TAMARI, M. (1980), "The financial structure of the small firm: an international comparison of corporate accounts in the United States, France, United Kingdom, Israël and Japan », American Journal of Small Business, vol. 4, n 4, p. 20-34.

TINIC, S.M. (1988), «Anatomy of initial public offerings of common stock», Journal of Finance, vol. 43, p. 789-822. 